\title{
Social media mining for smart cities and smart villages research
}

\author{
Miltiadis D. Lytras ${ }^{1,2}$ (1) $\cdot$ Anna Visvizi ${ }^{2,3}$ (D) Jari Jussila ${ }^{4}$ (I)
}

Published online: 8 June 2020

(C) Springer-Verlag GmbH Germany, part of Springer Nature 2020

\begin{abstract}
The imperative of well-being and improved quality of life in smart cities context can only be attained if the smart services, so central to the concept of smart cities, correspond with the needs, expectations and skills of cities' inhabitants. Considering that social media generate and/or open real-time entry points to vast amounts of data pertinent to well-being and quality of life, such as citizens' expectations, opinions, as well as to recent developments related to regulatory frameworks, debates, political decisions and policymaking, the big question is how to exploit the potential inherent in social media and use it to enhance the value added smart cities generate. Social mining is traditionally understood as the process of representing, analyzing, and extracting actionable patterns and trends from raw social media data. In the context of smart cities, this special issue focuses on how social media data, also potentially combined with other data, can be used to optimize the efficiency of city operations and services, and thereby contribute more efficiently to citizens' well-being and quality of life.
\end{abstract}

Keywords Soft computing $\cdot$ Smart cities $\cdot$ Smart villages $\cdot$ Social media mining $\cdot$ Well-being $\cdot$ Quality of life

\section{Introduction}

Research on smart cities is maturing, and new interdisciplinary approaches to the study of smart cities are being proposed (Visvizi and Lytras 2018). Social mining in smart cities and smart villages research is a one such new emerging research area. This special issue covers social mining in smart cities and smart villages from various perspectives.

The objective of this special issue is to shed light on recent advances in social mining and, considering, the growing dynamics of social media interaction, to seize the opportunity and to tap to the potential inherent in social

Miltiadis D. Lytras

miltiadis.lytras@gmail.com

Anna Visvizi

avisvizi@gmail.com

Jari Jussila

jari.jussila@gmail.com

King Abdulaziz University, Jeddah, Saudi Arabia

2 Effat University, Jeddah, Saudi Arabia

3 Deree College, The American College of Greece, Athens, Greece

4 Häme University of Applied Sciences, Hämeenlinna, Finland media. By so doing, the Editors of this Special Issue seek to encourage out-of-the box debate on how to make smart cities 'smarter,' i.e., more responsive to the needs and expectations of cities' inhabitants and more usable by them. As regulatory frameworks and policymaking strategies are key to the development of the context in which smart cities' can become 'smarter,' this Special Issue seeks also interdisciplinary approaches that bring together soft computing, smart cities research and regulatory/policymaking considerations.

Soft computing refers to a way of solving problems in a similar way to humans that are often imprecise but more effective than precise approaches, such as, conventional hard computing. Soft computing developed to complement precise and accurate hard computing approaches (Zadeh 1998). In contrast to hard computing, soft computing is tolerant of imprecision, uncertainty and partial truth (Zadeh 1994). However, Zadeh (1998) also points out that soft computing should be considered complementary and symbiotic rather than competitive and exclusive to hard computing approaches. The primary purpose of soft computing is to offer construct for modeling of imprecise and uncertain information by using approximate reasoning techniques to provide efficient and low-cost solutions (Mitra et al. 2002). 
Soft computing methodologies include fuzzy logic, neurocomputing, evolutionary computing and probabilistic computing (Zadeh 1998). Chaos theory tools and methodologies (Azar and Vaidyanathan 2016) overlap soft computing methodologies, and some authors (e.g., Gupta et al. 2020) also include them under the umbrella of soft computing. Fuzzy sets (Zadeh 1965) offer capabilities to deal with uncertain information, whereas rough sets (Pawlak 2012) and neural networks (Hornik et al. 1989) are used for extracting rules from the data and for classification tasks. Evolutionary computing refers to a family of algorithms that are inspired by biological evolution, such as genetic algorithms (Holland 1975), self-organizing maps (Kohonen 1997), swarm intelligence (Christian and Daniel 2008), etc. Evolutionary computing approaches are primarily used in optimization, for instance, genetic algorithms (Flockhart et al. 1996) are used in optimization tasks like query optimization and template selection (Mitra et al. 2002).

Soft computing methodologies, especially fuzzy logic, neural networks and genetic algorithms can be seen already quite established in many disciplines (Zadeh 1996; Lotfi and Garibaldi 2013); however, their application in social mining is relatively recent research area with still very few studies. Fuzzy granular theory has been used, for instance, for identifying fuzzy-rough communities (Pal et al. 2014; Kundu and Pal 2015a, b). Fuzzy social network analysis has been explored in Nair and Sarasamma (2007). In addition, fuzzy logic has also been explored for opinion mining (Bing and Chan 2014), for handling the uncertainty in sentiment analysis of big social data (Mukkamala et al. 2013; Mukkamala et al. 2014) and social set analysis of corporate social media crises on Facebook (Mukkamala et al. 2015; Jussila et al. 2017).

Topics of interest of the Special Issue include but not limited to:

- The use of soft computing approaches to extract actionable patterns and trends from social media to improve services and services' delivery to citizens

- Soft computing supporting crowdsourcing,

- Soft computing supporting civic engagement

- Soft computing in detecting trends in infrastructure and asset utilization and failures

- Novel methods for supporting smart city planning, design and construction

- Leveraging social media and internet of things (IoT) data to solve challenges specific to cities and urban areas

- Soft computing approaches to improve sustainability of smart cities

- Regulatory frameworks and social mining in the smart cities context

- Best practices

\section{Overview of the special issue}

The emerging domain of social computing for smart cities research is a dynamic multi-disciplinary area for theoretical and applied research. Disciplines including computer science and social sciences as well as sophisticated information processing capabilities provide a variety of innovative propositions for value adding services and platforms. In the wide range of theoretical considerations and computational intelligence, the following are some of the key aspects that were covered in our special issue:

\subsection{Concepts and ideas explored}

- Soft computing methods and algorithms

- Relation extraction of named entities in social media

- Cyberbullying and Violence detection over social media

- Question and Answering platforms

- Social media dynamics and User Similarities

- Social Trust

- Cultural identity

- Public sentiment and political situation analysis

The previous list is not exhaustive. Many other ideas and concepts are included in the theoretical domain of social mining for smart cities research. The recent pandemic for example of covid-19 is a good example on how the social mining can guide significant advanced data mining for high social value impact. The capacity of social mining to design and implement big data information processing provides a new fertile ground for sophisticated decision making. In a recent paper, AI-Youbi et al. (2020) analyze the impact of social mining for pandemic framework (Fig. 1).

\subsection{Computational intelligence and services}

The applied social mining research for smart cities and smart villages integrates multiple computational methods, including machine learning, semantic web and ontologies, soft computing methods, fuzzy logic, neural networks and advanced big data and analytics approaches. The following is an indicative and not exhaustive list.

- Soft computing methods and algorithms

- Simulation environments

- Formal modelling of OWL ontologies

- Artificial Intelligence innovations

- Altmetrics, Analytics and Key performance indicators for Social Computing

- Deep graph convolutional networks

- Machine learning algorithms 
Fig. 1 Theoretical concepts and ideas explored

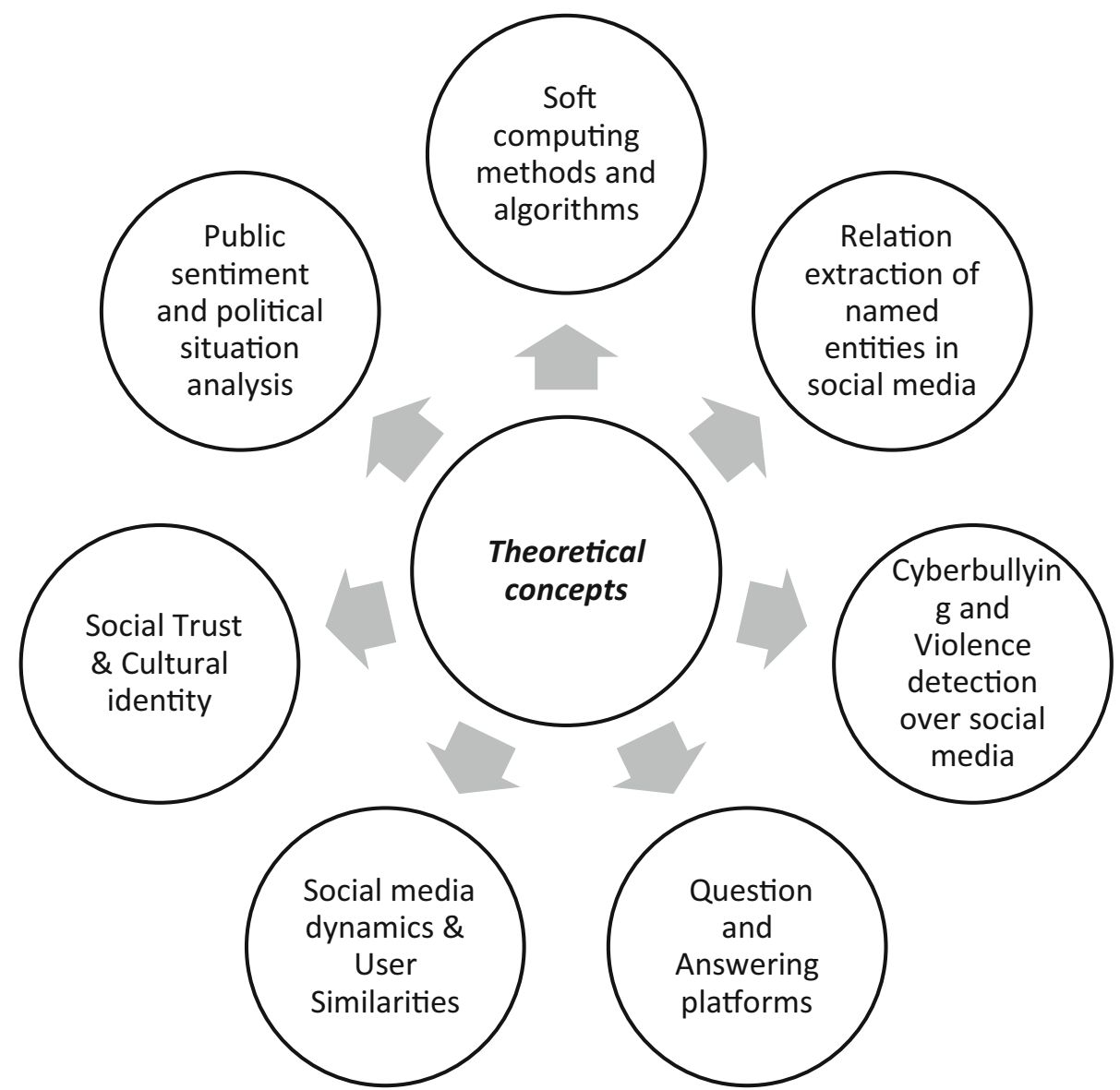

In Fig. 2, below, we provide a very abstract visualization for this context. It is our belief that in the near future social computing, and mining will evolve further as a key pillar for sustainability though data science.

In section below, we summarize the accepted papers.

\subsection{List of accepted papers}

- A soft computing approach to violence detection in social media for smart cities

- Research on relation extraction of named entity on social media in smart cities

- K-OpenAnswer: a simulation environment to analyze the dynamics of massive open online courses in smart cities

- Formal modelling of OWL ontologies-based requirements for the development of safe and secure smart city systems

- Unifying user similarity and social trust to generate powerful recommendations for smart cities using collaborating filtering-based recommender systems

- Towards Cyberbullying-free social media in smart cities: a unified multi-modal approach
- Quantification of cultural identity through artificial intelligence: a case study on the Waorani Amazonian ethnicity

- Bot prediction on social networks of Twitter in altmetrics using deep graph convolutional networks

- Predicting the helpfulness score of online reviews using convolutional neural network

- Predicting stock market trends using machine learning algorithms via public sentiment and political situation analysis

\section{Conclusions}

The domain of social mining for smart cities and smart villages research is a very dynamic research domain. Soon given the increased contributions of humans and businesses to diverse social media, the social mining ecosystem will increase significantly both in terms of available data and applications. Furthermore, the continuous discussion for the social impact of social impact of big data (Lytras and Visvizi 2019) will lead to more sophisticated social mining platforms for analyzing the social impact of big data applications and services. In this direction, the 
Fig. 2 Computational intelligence and services

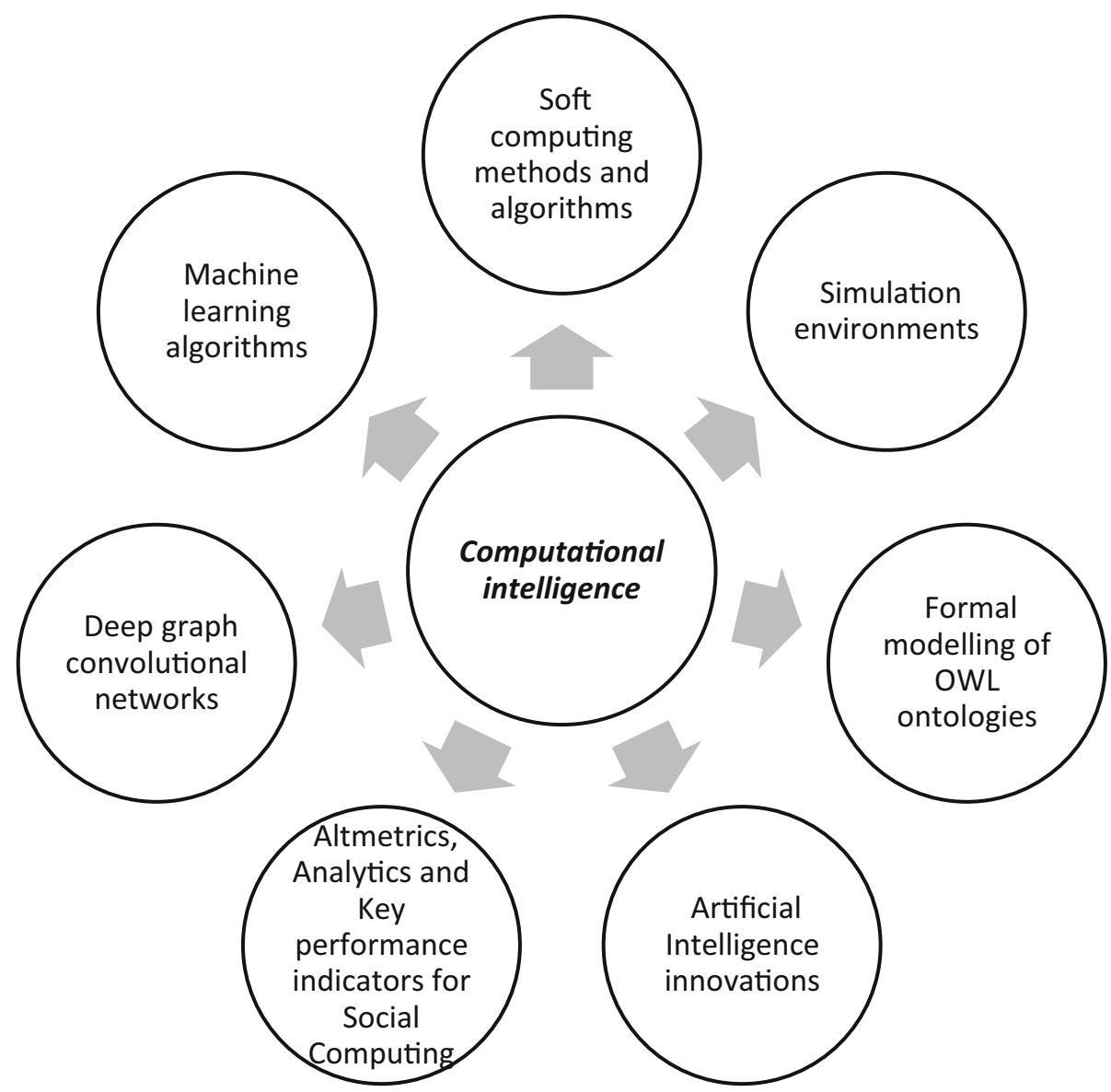

management of microcontents over social media will require annotation services capable of understanding similarities and identities (Alkmanash et al. (2019), Visvizi et al. (2020)).

On of the key conclusions of this special issue is that Future smart cities research (Lytras et al. 2020) will have a dominant social mining and social computing component.

We want to truly thank the Editor in Chief, the reviewers, the contributors and the editorial assistants for making this special issue possible. It is our distinct honor to serve the community of SOFT COMPUTING.

\section{Compliance with ethical standards}

Conflict of interest The authors declare that they have no conflict of interest.

\section{References}

Alkmanash EH, Jussila JJ, Lytras MD, Visvizi A (2019) Annotation of smart cities twitter microcontents for enhanced citizen's engagement. IEEE Access 99:1-1. https://doi.org/10.1109/ ACCESS.2019.2935186
Al-Youbi AO, Al-Hayani A, Bardesi HJ, Basheri M, Lytras MD, Aljohani NR (2020) The King Abdulaziz University (KAU) pandemic framework: a methodological approach to leverage social media for the sustainable management of higher education in crisis. Sustainability 12(11):4367

Azar AT, Vaidyanathan S (eds) (2016) Advances in chaos theory and intelligent control, vol 337. Springer, New York

Bing L, Chan KC (2014) A fuzzy logic approach for opinion mining on large scale twitter data. In: 2014 IEEE/ACM 7th international conference on utility and cloud computing. IEEE, pp 652-657

Christian B, Daniel M (2008) Swarm intelligence introduction and application. Natural Computing series. Springer, New York

Flockhart IW, Radcliffe NJ, Simoudis E, Han J, Fayyad U (1996) A genetic algorithm-based approach to data mining. In: KDD, pp 299-302

Gupta BB, Agrawal DP, Yamaguchi S et al (2020) Soft computing techniques for big data and cloud computing. Soft Comput 24:5483-5484. https://doi.org/10.1007/s00500-020-04766-2

Holland J (1975) Adaption in natural and artificial systems. University of Michigan Press, Ann Arbor

Hornik K, Stinchcombe M, White H (1989) Multilayer feedforward networks are universal approximators. Neural Netw 2(5):359-366

Jussila J, Menon K, Gupta J, Kärkkäinen H (2017) Who is who in big social data? A bibliographic network analysis study. In: Proceedings of the 4th European conference on social media, pp 161-169

Kohonen T (1997) Exploration of very large databases by selforganizing maps. In: Proceedings of international conference on neural networks (icnn'97), vol 1. IEEE, pp PL1-PL6 
Kundu S, Pal SK (2015a) FGSN: fuzzy granular social networksmodel and applications. Inf Sci 314:100-117

Kundu S, Pal SK (2015b) Fuzzy-rough community in social networks. Pattern Recogn Lett 67:145-152

Lotfi A, Garibaldi JM (eds) (2013) Applications and science in soft computing, vol 24. Springer, New York

Lytras MD, Visvizi A (2019) Big data and their social impact: preliminary study. Sustainability 11(18):5067

Lytras MD, Visvizi A, Torres-Ruiz M, Damiani E, Jin P (2020) IEEE access special section editorial: urban computing and well-being in smart cities: services, applications, policymaking considerations. IEEE Access 8:72340-72346. https://doi.org/10.1109/ ACCESS.2020.2988125

Mitra S, Pal SK, Mitra P (2002) Data mining in soft computing framework: a survey. IEEE Trans Neural Netw 13(1):3-14

Mukkamala RR, Hussain A, Vatrapu R (2013) Towards a formal model of social data. IT University of Copenhagen, Denmark, IT University Technical Report Series TR-2013-169, 1(3), p 5

Mukkamala RR, Hussain A, Vatrapu R (2014) Fuzzy-set based sentiment analysis of big social data. In: 2014 IEEE 18th international enterprise distributed object computing conference. IEEE, pp 71-80

Mukkamala RR, Sørensen JI, Hussain A, Vatrapu R (2015) Social set analysis of corporate social media crises on facebook. In: 2015 IEEE 19th international enterprise distributed object computing conference. IEEE, pp 112-121

Nair PS, Sarasamma ST (2007) Data mining through fuzzy social network analysis. In: NAFIPS 2007-2007 annual meeting of the north american fuzzy information processing society. IEEE, pp 251-255
Pal SK, Kundu S, Murthy CA (2014) Centrality measures, upper bound, and influence maximization in large scale directed social networks. Fund Inf 130(3):317-342

Pawlak Z (2012) Rough sets: theoretical aspects of reasoning about data, vol 9. Springer, New York

Visvizi A, Lytras MD (2018) It's not a fad: Smart cities and smart villages research in European and global contexts. Sustainability 10:2727. https://doi.org/10.3390/su10082727

Visvizi A, Jussila J, Lytras MD, Ijäs M (2020) Tweeting and mining OECD-related microcontent in the post-truth era: a cloudbased app. Comput Hum Behav 107:105958. https://doi.org/10.1016/j. chb.2019.03.022

Zadeh LA (1965) Fuzzy sets. Inf Control 8(3):338-353. https://doi. org/10.1016/S0019-9958(65)90241-X

Zadeh LA (1994) Fuzzy logic, neural networks, and soft computing. Commun ACM 37(3):77-84

Zadeh LA (1996) Fuzzy logic, neural networks, and soft computing. Fuzzy sets, fuzzy logic, and fuzzy systems: selected papers by Lotfi A. Zadeh. World Scientific Publishing Co., Inc., USA, pp 775-782

Zadeh LA (1998) Some reflections on soft computing, granular computing and their roles in the conception, design and utilization of information/intelligent systems. Soft Comput 2(1):23-25

Publisher's Note Springer Nature remains neutral with regard to jurisdictional claims in published maps and institutional affiliations. 